\title{
Colonic duplication presenting as a huge abdominal mass in an adult female
}

\author{
Kung-Chuan Cheng ${ }^{1} \cdot$ Sheung-Fat Ko ${ }^{2} \cdot K$-Chao Lee ${ }^{1}$ (D)
}

Accepted: 16 September 2019 / Published online: 23 October 2019

(C) The Author(s) 2019

\begin{abstract}
Introduction Duplications of the alimentary tract are a rare congenital malformation. Most of the cases are symptomatic and diagnosed before 2 years of age. Here, we report a young female presented with a huge abdominal mass, and colonic duplication was confirmed during laparotomy.

Case presentation A 29-year-old female had chronic constipation treated with laxative agents. She presented to the emergency room with abdominal cramping for 3 days, accompanied with intermittent fever and vomiting. A huge movable abdominal mass was noted during physical examination. Computerized tomography showed a long segmental dilated bowel lumen with stool impaction and bowel wall thickening of the dilated lumen in the left abdomen, highly suggestive of a long tubular colon duplication. The patient underwent subtotal colectomy. Specimen subsequently confirmed the diagnosis for colonic duplication from cecum to sigmoid colon, and the duplicated colon was found on the antimesenteric side of the native colon. She had a stable postoperative course and was discharged 9 days later.

Discussion Duplications of the alimentary tract are a rare congenital anomaly. Colonic duplication is an even more unusual malformation of this type. It can be classified into cystic or tubular type according to the gross morphology and may or may not be associated with other congenital anomalies. Most common presentation includes abdominal distention, refractory constipation, and bowel obstruction like many other colorectal conditions. Thus, its indistinct symptoms make it difficult to be diagnosed preoperatively. The recommended treatment is surgical resection of the duplicated lumen along with the attached native colon.
\end{abstract}

Keywords Colonic duplication · Adult · Tubular type

\section{Introduction}

Duplications of gastrointestinal tract are rare congenital diseases and are usually diagnosed in childhood. The diagnosis can be challenging in adulthood because of vague abdominal symptoms. We report a case of a young female with a tubulartype colonic duplication from the cecum to the sigmoiddescending colon junction presenting to our emergency room as a huge abdominal mass.

Ko-Chao Lee

kmch4329@gmail.com

1 Division of Colorectal Surgery, Department of Surgery, Chang Gung Memorial Hospital - Kaohsiung Medical Center, Chang Gung University College of Medicine, Kaohsiung, Kaohsiung Taiwan

2 Department of Radiology, Chang Gung Memorial Hospital Kaohsiung Medical Center, Chang Gung University College of Medicine, Kaohsiung Taiwan

\section{Case presentation}

A 29-year-old woman presented to our emergency department with a huge abdominal mass, accompanied by abdominal cramping, vomiting, and fever for 3 days. She had had chronic constipation since childhood. Prior barium lower gastrointestinal study performed 9 years ago reported the presence of markedly dilated colon with excessive fecal material retention and initial impressions of Hirschsprung's disease or functional constipation were made. The patient refused further assessment with colonoscopy for tissue proof. She was conservatively managed with laxative agents and subsequently lost follow-up for 9 years until the present visit to our emergency department. During this period, she was treated as chronic constipation with various laxatives at multiple institutions.

The patient's vital signs on arrival were unremarkable except for tachycardia with a pulse rate of 144 beats per minute. Physical examination showed a huge movable mass at the left side of her abdomen with mild tenderness. Laboratory data 
were within normal limits except for elevation of the level of C-reactive protein $(175 \mathrm{mg} / \mathrm{dL})$. Contrast-enhanced abdominal computed tomography (CT) (Fig. 1a and b) for assessment of palpable abdominal mass showed a long segmental dilated bowel lumen with stool impaction and bowel wall thickening of the dilated lumen in the left abdomen. Part of the ascending colon and medially displaced descending colon close to the dilated lumen were suspected but the exact anatomic details were hard to confirm. Moreover, after correlation with prior barium enema images (Fig. 2), a collapsed native colon closely apposing the dilated lumen with partial barium filling was identified, highly suggestive of a long tubular colon duplication.

The imaging findings were verified in subsequent laparotomy and a long segmental colonic duplication at the antimesenteric side sharing the same blood supplies with the native colon from proximal ascending to sigmoid-descending colon junction level were revealed. Communicating channel at the transverse colon between native and duplicated colons
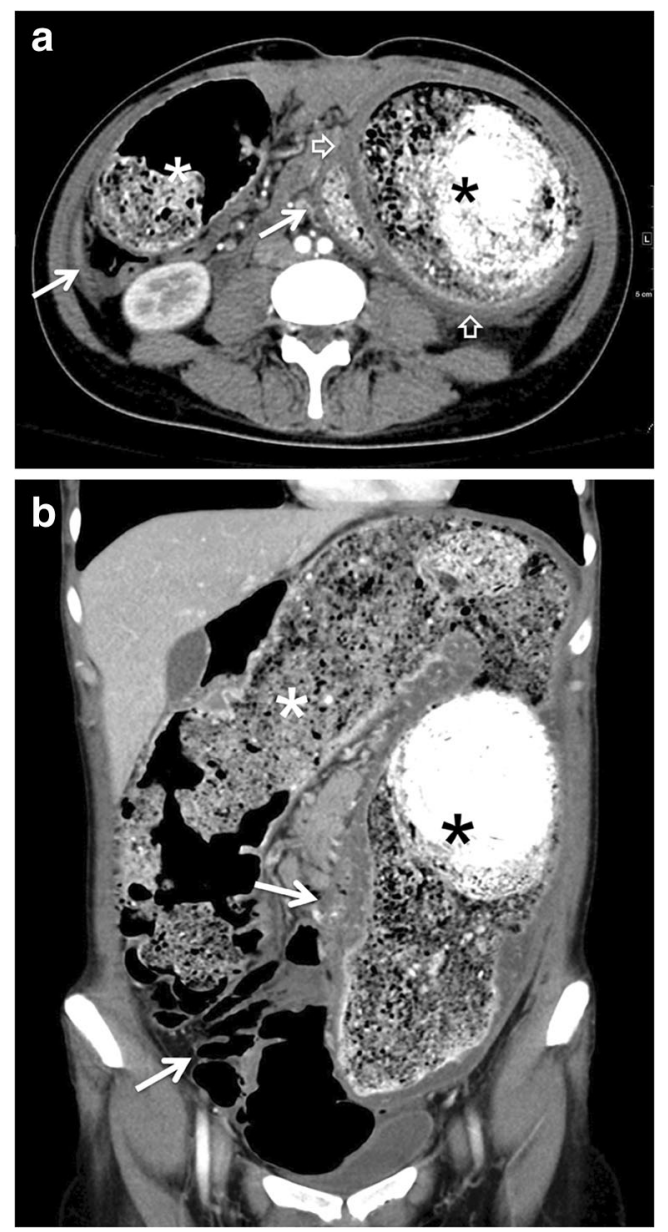

Fig. 1 Axial (a) and coronal (b) abdominal CT images show a long segmental dilated bowel lumen with stool impaction (asterisk) and focal bowel wall thickening (open arrows) in the left side of the abdomen. Part of the ascending colon and descending colon (arrows) closely abutting the dilated bowel lumen is suspected

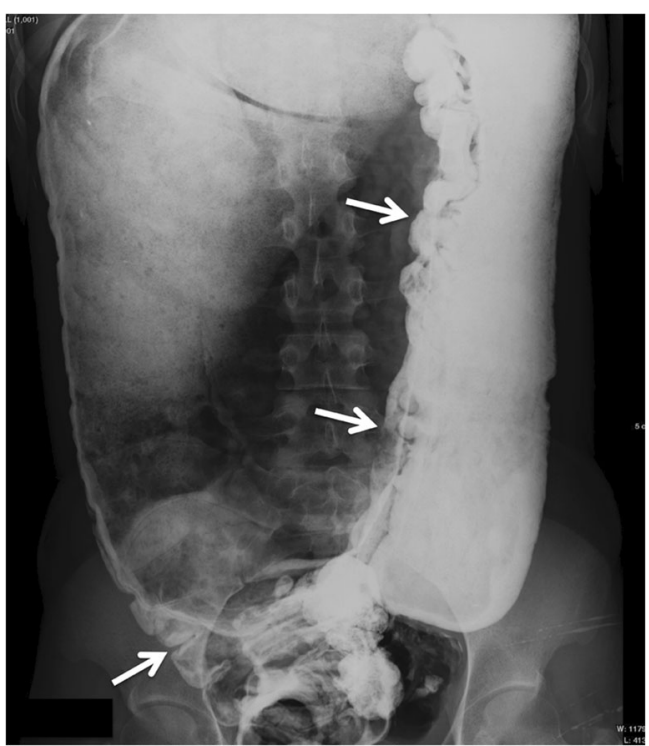

Fig. 2 Barium enema of colon performed 9 years prior to this admission was reviewed. On 24-h follow-up study, partial filling of barium into the dilated bowel lumen was found. Of note, part of proximal ascending colon and partially collapsed descending colon (arrows) adjacent to the dilated bowel lumen were identified, highly suggestive of long segmental colon duplication with marked fecal material retention

was noted. In addition, concurrent ischemic changes at the duplicated descending colon wall were also found. Therefore, a subtotal colectomy was performed (Fig. 3). The final diagnosis of tubular colonic duplication was confirmed by histopathological examinations with demonstration of typical colon mucosa in both native and duplicated lumens (Fig. 4) as well as ischemic changes with inflammation in duplicated descending colon. Figure 5 demonstrates the gross anatomy finding of this patient. The patient recovered uneventfully and was discharged on the 9th day after the operation.

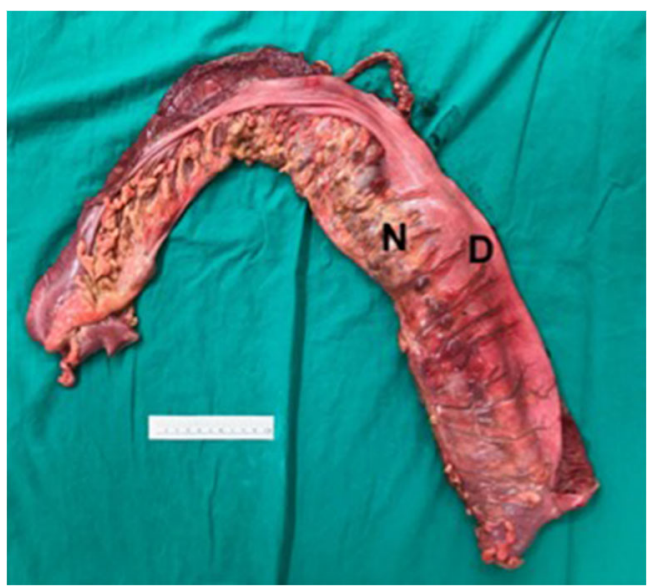

Fig. 3 Specimen confirmed the diagnosis of colonic duplication from the cecum to the sigmoid colon. The duplicated colon (D) was found on the antimesenteric side of the native colon $(\mathrm{N})$ 


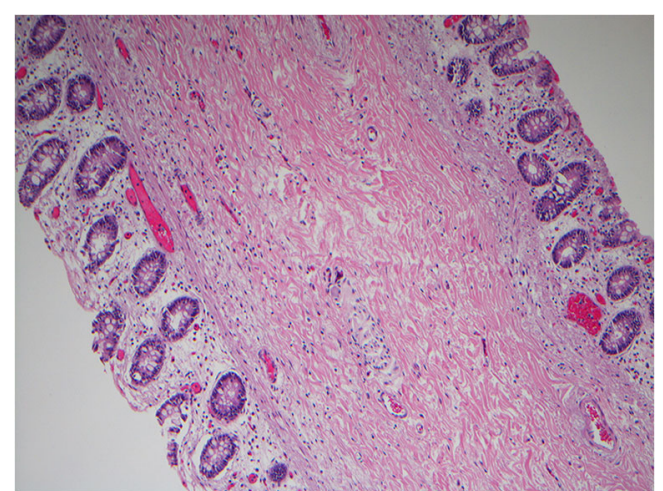

Fig. 4 The septa between two lumens were lined by colonic mucosa on both sides

\section{Discussion}

Duplications of the gastrointestinal tract are rare. These anomalies can develop anywhere along with the alimentary tract and are most commonly found in the small intestine. Colonic duplication comprises $6.8 \%$ of all gastrointestinal duplications [1]. Pathologically, duplications of GI tract are defined as (1) a microscopic structure resembling the normal bowel, having mucosa, muscle layers, and serosa; (2) lining with a mucosa similar to some portion of the alimentary tract; and (3) lesions that are contiguous with some portion of the intestinal tract [2]. Gastrointestinal duplications can be classified as cystic (spherical) or tubular according to their gross morphologies. The tubular type of gastrointestinal duplication contributes to less than $20 \%$ of cases. Such lesions can present in T-shape or Y-shape and are frequently associated with concurrent genitourinary anomalies or colovesical fistula [3]. The pathogenesis of this disease is not fully understood. Several theories have been proposed for the development of gastrointestinal duplications but none can solely explain all the locations where this anomaly may occur. Moreover, a great variety

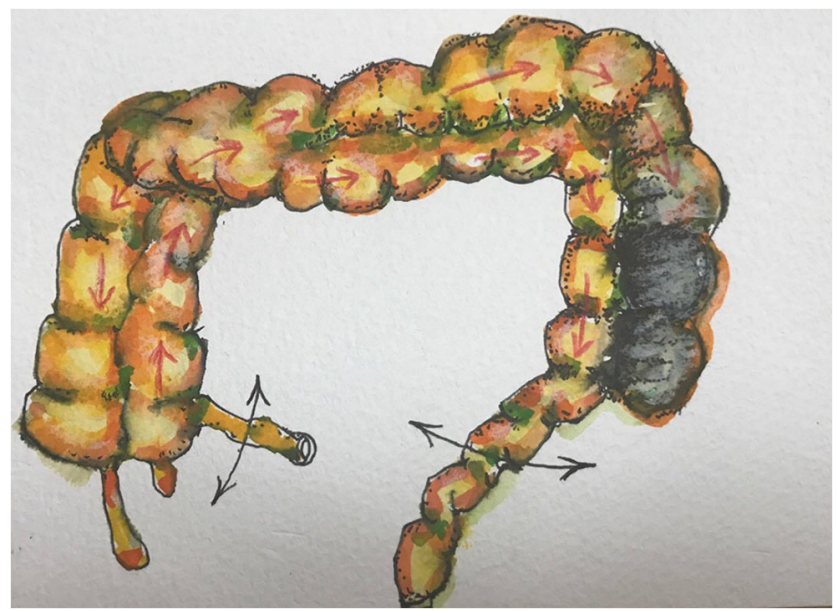

Fig. 5 The drawing demonstrates the gross anatomical finding of this patient of explanations suggestive of the development of this entity may be multifactorial [4].

Most patients with colonic duplication are diagnosed before the age of two. The most common clinical presentation of colonic duplications is intestinal obstruction due to compression of the native colon by dilated blind-ended duplicated lumen, with other symptoms including constipation, vomiting, volvulus, and perforation [5]. Since the abdominal symptoms are vague, some patients with indolent symptoms may not be detected until their adulthood. In fact, if without other associated malformations, most adult patients remain undiagnosed unless complications develop.

Owing to non-specific and a wide variety of abdominal symptoms, the diagnosis of colonic duplication could be very challenging. Though plain abdominal radiograph and ultrasonography might be useful, the diagnosis is best established by abdominal CT. Colonoscopy is not helpful unless the ostium between duplication and the native lumen is large enough to be detected [3]. Despite the availability of various diagnostic tools, the preoperative diagnosis of colonic duplication was made in only one out of seven cases in a case series study [6].

The management of asymptomatic colonic duplication is not well established. Some authors suggest that surgical resection should be reserved for symptomatic causes, though others suggest resection once the diagnosis is made to prevent further complications and malignant change [6, 7]. For symptomatic patients, on the other hand, most authors recommend en-bloc resection together with the normal colon because the duplicated colon always shares the same mesenteric blood supply with the normal colon. Either laparotomy or minimally invasive surgery can be considered according to the patient's clinical presentation. Prognosis is good owing to the benign nature of the disease.

Here, we report a very rare tubular type of colonic duplication without any genitourinary or other congenital defect in an adult, presenting with a huge abdominal mass initially. Surgical specimen showed a long segmental tubular duplication from the cecum to the sigmoid-descending colon junction, with a huge communication between the native and duplicated lumen at the transverse colon. The bowel continuity was reconstructed without diverting the stoma, and the patient recovered well from the operation.

Open Access This article is distributed under the terms of the Creative Commons Attribution 4.0 International License (http:// creativecommons.org/licenses/by/4.0/), which permits unrestricted use, distribution, and reproduction in any medium, provided you give appropriate credit to the original author(s) and the source, provide a link to the Creative Commons license, and indicate if changes were made.

\section{References}

1. Puligandla PS, Nguyen LT, St-Vil D, Flageole H, Bensoussan AL, Nguyen VH, Laberge JM (2003) Gastrointestinal duplications. J 
Pediatr Surg 38(5):740-744. https://doi.org/10.1016/jpsu.2003. 50197

2. Gross RE, Holcomb GW, Farber S (1952) Duplications of the alimentary tract. Pediatrics 9(4):448-468 http://www.ncbi.nlm.nih.gov/ pubmed/14920118

3. Al-Jaroof AH, Al-Zayer F, Meshikhes A-WN (2014) A case of sigmoid colon duplication in an adult woman. BMJ Case Reports 2014: 1-4. https://doi.org/10.1136/bcr-2014-203874

4. Castejón-Casado J, Muñoz Miguelsanz M, Diaz E, Gomez M, Padilla Garcia M, Valade R (2014) Acute abdomen secondary to complete tubular colonic duplication. J Indian Assoc Pediatr Surg 19(3):166. https://doi.org/10.4103/0971-9261.136477

5. Kekez T, Augustin G, Hrstic I, Smud D, Majerovic M, Jelincic Z, Kinda E (2008) Colonic duplication in an adult who presented with chronic constipation attributed to hypothyroidism. World $\mathrm{J}$ Gastroenterol 14(4):644-646. https://doi.org/10.3748/wjg.13.644

6. Mourra N, Chafai N, Bessoud B, Reveri V, Werbrouck A, Tiret E (2010) Colorectal duplication in adults: report of seven cases and review of the literature. J Clin Pathol 63(12):1080-1083. https:// doi.org/10.1136/jcp.2010.083238

7. Fotiadis C, Genetzakis M, Papandreou I, Misiakos EP, Agapitos E, Zografos GC (2005) Colonic duplication in adults: report of two cases presenting with rectal bleeding. World J Gastroenterol 11(32):5072-5074. https://doi.org/10.3748/wjg.v11.i32.5072

Publisher's note Springer Nature remains neutral with regard to jurisdictional claims in published maps and institutional affiliations. 